\title{
COMPETÊNCIA SOCIAL, INCLUSÃO ESCOLAR E AUTISMO: REVISÃO CRÍTICA DA LITERATURA ${ }^{1}$
}

\author{
Síglia Pimentel Höher Camargo e Cleonice Alves Bosa \\ Universidade Federal do Rio Grande do Sul, Porto Alegre, Brasil
}

\begin{abstract}
RESUMO: O autismo se caracteriza pela presença de um desenvolvimento acentuadamente atípico na interação social e comunicação, assim como pelo repertório marcadamente restrito de atividades e interesses. Estas características podem levar a um isolamento contínuo da criança e sua família. Entretanto, acredita-se que a inclusão escolar pode proporcionar a essas crianças oportunidades de convivência com outras da mesma faixa etária, constituindo-se num espaço de aprendizagem e de desenvolvimento da competência social. O objetivo deste estudo foi revisar criticamente a literatura a respeito do conceito de competência social e dos estudos atualmente existentes na área de autismo e inclusão escolar. Identificaram-se poucos estudos sobre este tema, os quais apresentam limitações metodológicas. Este panorama aponta para a necessidade de investigações que demonstrem as potencialidades interativas de crianças com autismo e a possibilidade de sua inclusão no ensino comum, desde a educação infantil.
\end{abstract}

PALAVRAS-CHAVE: competência social; autismo; inclusão escolar.

\section{SOCIAL COMPETENCE, SCHOOL INCLUSION AND AUTISM: CRITICAL LITERATURE REVIEW}

ABSTRACT: Autism is a condition characterized by an atypical development in the social interaction and communication, and by a remarkably restricted repertoire of activities and interests. These characteristics can lead the child and her family to a continuous isolation. However, it is believed that the school inclusion can provide these children opportunities to be with others of the same age group, allowing the development of the social competence and learning. The aim of this study was to critically review the literature on the concept of social competence and school inclusion in the area of autism. Few studies were identified about this topic, but with methodological limitations. This situation points to the need of studies that demonstrate the autistic children's interactive potentialities and the possibility of their inclusion in the mainstream school, since early education.

KEYWORDS: social competence; autism; school inclusion.

O interesse nas questões da interação social e as reflexões sobre a sua importância para o comportamento humano surgiram no século passado. Entre 1830 e 1930 já era possível encontrar uma ampla e variada produção que pressupunha que as relações sociais interpessoais se encontravam entre os principais determinantes da natureza humana, sendo passíveis de investigação científica (Aranha, 1993; Dessen \& Aranha, 1994). Já naquela época, apontava-se, inclusive, para a importância da experiência social com pares (Hartup, 1983). Entretanto, as ideias geradas naquele período possuíam um caráter mais especulativo, pois ainda não havia sido construída uma base empírica consistente e métodos sistemáticos para a coleta dos dados nessa área. Foi somente a partir da década de 30 que se desenvolveram métodos e técnicas de observação de grupo, em especial os instrumentos sociométricos.

Igualmente envolvido com a temática das relações entre indivíduo e sociedade no mesmo período, George Herbert Mead dedicou-se à investigação da gênese do eu humano no processo da interação social. $\mathrm{Na}$ abordagem denominada por seus seguidores de interacionismo simbólico, Mead (1934/1972) foi um dos fundadores da sociologia empírica e sistemática, sendo um dos primeiros a descrever a socialização como construção de uma identidade social na e pela interação com os outros (Dubar, 1999). Para esse teórico, o centro do processo de socialização é a comunicação pelo gesto, que constitui uma adaptação à reação do outro. Tais gestos são atos parciais dirigidos a outros, os quais devem receber e responder a eles. Assim, o gesto é uma ação incompleta, cuja complementação e sentido são construídos apenas na interação com os outros. Esses "outros", a quem Mead chamou de "outros significativos", são os agentes da socialização, constituídos pelos indivíduos que possuem uma importância significativa na adaptação da criança ao mundo em que ela vive. Desse modo, o processo de socialização está na base da construção do $\mathrm{Eu}$, dada pela mediação dos outros e suas respostas. 
A partir dos anos 30, entretanto, estudos sobre as questões da interação social são praticamente inexistentes, sendo retomados somente após a Segunda Guerra Mundial, com uma notável ênfase na relação mãe-criança. Conforme Pedrosa e Carvalho (2005), o estudo da interação entre pares foi relegado até a década de 70 e, dada a centralidade na interação pais-filhos, grande parte dos psicólogos considerava o relacionamento entre iguais como menos importante. Na década de 70, portanto, a retomada pelo interesse no estudo das relações sociais gera a produção de diversos trabalhos e propostas teóricas quanto a sua natureza e função. Nesse sentido, "no estudo do desenvolvimento humano, a interação social tem ocupado diferentes espaços, dependendo da função a ela atribuída por diferentes abordagens teóricas" (Aranha, 1993, p.19). Entretanto, parece haver um consenso entre elas no sentido de que o sucesso da constituição psíquica do indivíduo depende, primordialmente, do processo de socialização. É no contexto das relações sociais que emergem a linguagem, o desenvolvimento cognitivo (Moura, 1993), o autoconhecimento e o conhecimento do outro. Além de proporcionar outros conhecimentos sobre o mundo, a interação social atua como precursora de relacionamentos subsequentes (conjugal e parental).

$\mathrm{Na}$ área da Psicologia do Desenvolvimento, Hartup (1989) aprofunda-se no estudo das interações sociais com pares, influenciado pelos paradigmas da cognição social (Piaget), aprendizagem social (Bandura) e as teorias sociogenéticas de Baldwin e Vigotsky. Esse autor sugere que toda criança necessita vivenciar dois tipos de relacionamentos: vertical e horizontal. O primeiro se caracteriza por relacionamentos complementares que envolvem apego a uma pessoa com maior poder social ou conhecimento, como os pais, a professora ou um irmão mais velho. Por outro lado, os relacionamentos horizontais são recíprocos e igualitários, pois envolvem companheiros da mesma idade, cujo poder social e comportamento mútuo se originam de um mesmo repertório de experiências. Esses dois tipos de relacionamento exercem funções diferentes para a criança e são necessários para o desenvolvimento de habilidades sociais efetivas. Enquanto a relação vertical proporciona segurança e proteção, cria modelos internos básicos e desenvolve habilidades sociais fundamentais, a relação horizontal desenvolve habilidades sociais que só podem ser experienciadas no relacionamento entre iguais: formas específicas de cooperação, competição e intimidade (Hartup, 1989, 1992).

Almeida (1997), ao estudar a relação entre crianças em idade escolar a partir da perspectiva de Hartup, afirma que a interação com pares não fornece apenas as experiências necessárias ao desenvolvimento de competências sociocognitivas, mas constitui-se em uma base fundamental para o autoconhecimento e para a compreensão do "self". Uma das premissas básicas das ideias de Hartup é de que a competência social é, em sua maior parte, aprendida com os companheiros.

A interação com outras crianças da mesma faixa etária proporciona contextos sociais que permitem vivenciar experiências que dão origem à troca de ideias, de papéis e o compartilhamento de atividades que exigem negociação interpessoal e discussão para a resolução de conflitos. No grupo de pares emergem as regras que estruturam as atividades de cooperação e competição. Desse modo, a qualidade das interações com iguais e a competência social influenciam-se mutuamente. É, sobretudo, através da investigação sobre as relações entre pares que se evidenciam as diferenças individuais na competência social (Almeida, 1997). Dessa forma, os companheiros representam uma fonte de relações imprescindível, provendo um contexto adicional único e poderoso que influencia as diferenças individuais durante o desenvolvimento social de qualquer criança (Castro, Melo, \& Silvares, 2003).

O conceito de competência social tem sido frequentemente utilizado como sinônimo de habilidades sociais. Embora sejam conceitos intimamente relacionados, Del Prette e Del Prette $(1996,1999)$ fazem a distinção entre ambos. Para esses autores, a habilidade social possui um caráter descritivo, o qual se refere à totalidade dos desempenhos do indivíduo perante as demandas de uma situação em sentido amplo. Já a competência social é entendida como um julgamento sobre a qualidade da performance individual em uma determinada situação. Assim, é possível afirmar que crianças com maiores habilidades sociais sejam consideradas socialmente mais competentes. Nesse sentido, a competência social é, de um modo geral, um construto psicológico que reflete múltiplas facetas do funcionamento cognitivo, emocional e comportamental. Trata-se, portanto, não de um traço global da personalidade, mas um conjunto de comportamentos aprendidos no decurso das interações sociais, sobretudo, na interação com pares. Almeida (1997) afirma que o desenvolvimento da competência social, numa perspectiva do desenvolvimento organizacional e relacional (Waters \& Sroufe, 1983), pretende enfatizar a diversidade de soluções adaptativas que permitem à criança desenvolver-se socialmente, numa variedade de contextos e situações. Nesse sentido, as expressões da competência social podem assumir formas bastantes "impopulares", e o aparente desajuste pode não ser visto apenas como um déficit de aptidões, mas a necessidade de uma adequação ao contexto. Assim, uma concepção da competência social, enquanto construto desenvolvimental, deve enfatizar em que medida os comportamentos manifestos pela criança, num determinado período e contexto, representam 
soluções adaptativas do ponto de vista do seu nível de desenvolvimento. Esse princípio organizador estabelece que a avaliação da competência social se deva reportar a estes indicadores desenvolvimentais.

$\mathrm{Na}$ literatura encontram-se diversas perspectivas teóricas que privilegiam diferentes facetas bastante específicas da competência social. Entretanto, a principal crítica apontada à maioria dessas definições é que elas privilegiam a dimensão exteriorizável do comportamento social, desconsiderando o valor adaptativo do comportamento no ciclo de vida. Nesse sentido, Almeida (1997) chama a atenção para a importância de se compreender a competência social numa perspectiva desenvolvimental e contextualizada no seu espaço sociocultural. Segundo Waters e Sroufe (1983), a competência social é a capacidade de utilizar os recursos ambientais e pessoais para conseguir um "bom resultado desenvolvimental" em longo prazo, ou seja, a capacidade de ajustamento e saúde mental na idade adulta. Em curto prazo, os resultados são avaliados pelas consequências positivas que a criança retira do funcionamento adequado aos parâmetros estabelecidos para cada nível desenvolvimental, bem como a preparação para as tarefas desenvolvimentais do nível seguinte. Assim, a importância que assume a relação entre pares, dada a sua intensidade e permanência ao longo do desenvolvimento, torna inseparáveis o desenvolvimento da competência social e o das relações interpessoais.

Visto que os relacionamentos entre crianças da mesma idade desempenham um papel fundamental no desenvolvimento das habilidades sociais, a qualidade da convivência com estes "outros significativos" afeta positiva ou negativamente as diversas aquisições que delas se originam (Del Prette \& Del Prette, 2006; Hartup, 1996; Pepler \& Craig, 1998). Segundo Shaffer (1996), diversos estudos têm demonstrado as consequências "indesejáveis", resultantes da falta de amizade ou dificuldades nas relações com pares, tais como maior probabilidade de desenvolver problemas emocionais, ser menos sociável, apresentar ajustamento escolar mais pobre, obter menos ganhos educacionais, ser menos altruísta, entre outros. Além disso, evidências empíricas a partir de estudos longitudinais demonstram que há uma correlação entre dificuldades precocemente identificadas nas relações entre pares durante a infância e comportamento antissocial, abandono escolar (Gronlund \& Holmlund, 1958; Parker \& Asher, 1987) e, até mesmo, comportamentos delinquentes na adolescência e idade adulta (Farrington, Gallagher, Morley, Ledger, \& West, 1986; Olwes, 1980). Conforme Almeida (1997), crianças que são rejeitadas por seus pares, em comparação àquelas que são bem aceitas no grupo, têm maior probabilidade de desenvolver dificuldades socioemocionais. Assim, os relacionamentos entre pares proveem uma importante janela para o funcionamento social das crianças e são experiências críticas, ao longo da infância e da adolescência, no desenvolvimento de comportamentos adaptativos.

Desse modo, pode-se perceber uma tendência nos últimos anos em atribuir à interação social um papel importante no desenvolvimento da criança, enquanto via de formação de relações sociais (Dessen \& Aranha, 1994). Ao longo desses anos, as teorias do desenvolvimento social (etológica, piagetiana, da aprendizagem social, sistemas dinâmicos, sociointeracionista, sóciohistórica, dentre outros), apesar das diversidades na abordagem teórica e metodológica, são unânimes em reiterar que a interação social é a condição de construção do indivíduo e base do desenvolvimento do ser humano. Além disso, diversos estudos identificam a importância da interação com pares para o desenvolvimento da criança pré-escolar e de sua competência social (Almeida, 1997; Hartup, 1996). Estudos desenvolvidos por Lordelo e Carvalho (1998) e Oliveira e Rosseti-Ferreira (1993) com crianças pequenas em creche identificaram notáveis ganhos no desenvolvivento social dessas crianças, a partir da oportunidade de interação com pares, proporcionada pelo ambiente. Corsaro (1997), a partir da noção da reprodução interpretativa, na área da sociologia, também aponta a importância dos relacionamentos sociais para a criança. Para o autor, culturas infantis emergem na medida em que as crianças, interagindo com os adultos e com seus pares, tentam atribuir sentido ao mundo em que vivem. A cultura de pares é fundamental para a criança, pois lhe permite apropriar, reinventar e reproduzir o mundo que a rodeia.

Entretanto, o desenvolvimento social de algumas crianças já se encontra em risco desde os primeiros anos de vida, sendo o autismo o protótipo desses casos. Nesse sentido, a escola possui papel fundamental nos esforços para ultrapassar os déficits sociais dessas crianças, ao possibilitar o alargamento progressivo das experiências socializadoras, permitindo o desenvolvimento de novos conhecimentos e comportamentos.

\section{Autismo e Inclusão escolar}

O autismo é classificado pelo DSM-IV-TR (Associação Psiquiátrica Americana [APA], 2002) como um transtorno global do desenvolvimento, que se caracteriza pelo desenvolvimento acentuadamente atípico na interação social e comunicação e pela presença de um repertório marcadamente restrito de atividades e interesses. Os comprometimentos nessas áreas estão presentes antes dos três anos de idade, quando os pais, em geral, já percebem e preocupam-se com as limitações observadas, cada vez mais aparentes ao longo do desenvolvimento. Desse modo, observa-se uma 
dificuldade qualitativa de relacionar-se e comunicar-se de maneira usual com as pessoas, desde cedo na vida. Estudos epidemiológicos apresentam uma prevalência de aproximadamente $1 \mathrm{em}$ cada 200 indivíduos (Klin, 2006), sendo esta quatro vezes maior em meninos do que em meninas. Somente no Brasil, embora não existam dados epidemiológicos estatísticos, é estimado pela Associação Brasileira de Autismo que aproximadamente 600 mil pessoas apresentam essa síndrome (Bosa \& Callias, 2000), sem contar aqueles que não se enquadram em sua forma típica.

Quanto à etiologia, desde as primeiras considerações feitas por Kanner, em 1943, muitas reformulações nos mecanismos explicativos foram realizadas, sem, entretanto, chegar-se a conclusões consistentes. Isso pode ser observado nas diversas abordagens que historicamente tentaram estabelecer um lugar na dicotomia inato $\mathrm{x}$ ambiental de onde se possa definir o autismo. No entanto, a tendência atual na definição de autismo é a de conceituá-lo como uma síndrome comportamental, de etiologias múltiplas, com intensas implicações para o desenvolvimento global infantil (Volkmar, Lord, Bailey, Schultz, \& Klin, 2004). Embora ainda não haja um marcador biológico definitivo, alguns achados têm demonstrado particularidades nas áreas da genética e do funcionamento cerebral (Gupta \& State, 2006).

$\mathrm{O}$ autismo pode ocorrer em qualquer classe social, raça ou cultura, sendo que cerca de 65 a $90 \%$ dos casos estão associados à deficiência mental (Gadia, Tuchman, $\&$ Rotta, 2004). Essa incidência vem contra a noção estereotipada, derivada das descrições clássicas, de que crianças autistas possuem uma inteligência secreta e superior. Crianças com autismo de alto funcionamento (perfil cognitivo diferenciado em algumas das áreas de testes padronizados) representam apenas $30 \%$ dos casos diagnosticados (Bosa, 2002). Desse modo, é possível perceber a notável variação na expressão de "sintomas" do autismo. Crianças com funcionamento cognitivo mais baixo geralmente tendem a ser mudas e isoladas. Em outro nível, a criança pode aceitar passivamente a interação, mas raramente a procura, enquanto em um funcionamento mais alto é possível que a criança se interesse pela interação, mas o faz de modo bizarro (Klin, 2006). De qualquer modo, destaca-se que a noção de uma criança não-comunicativa, isolada e incapaz de mostrar afeto não corresponde às observações atualmente realizadas. De acordo com Bosa (2002), a ausência de respostas das crianças autistas deve-se, muitas vezes, à falta de compreensão do que está sendo exigido dela, ao invés de uma atitude de isolamento e recusa proposital. Nesse sentido, julgar que a criança é alheia ao que acontece ao seu redor restringe a motivação para investir na sua potencialidade para interagir. Lord e Magil (1989) já questionavam até que ponto o retrai- mento social das crianças com autismo não resultaria da falta de oportunidades oferecidas, mais do que algo inerente à própria síndrome. Almeida (1997) parte da noção de que o ser humano está inatamente programado para estabelecer vínculos sociais, mas que o desenvolvimento social só se constrói na sequência de interações, em qualquer estágio da vida. Conforme Cairns (1986), a trajetória individual não é determinada somente pelos componentes genéticos, nem somente nos primeiros anos de vida (embora seja este um período crítico para o desenvolvimento). É, sobretudo, determinada pelas condições em que se desenrola o desenvolvimento, incluindo as mudanças biológicas esperadas, os sistemas de relações sociais que envolvem a criança, as condições dos ambientes que circula, o nível cognitivo da criança, entre outros. Isso quer dizer que não se pode afirmar que no nível da intervenção não se possa minimizar as dificuldades que caracterizam qualquer síndrome. Um exemplo clássico é o caso de Victor, o menino "selvagem" capturado nas florestas do sul da França, há cerca de 200 anos. Avaliado em Paris pelo célebre psiquiatra Pinel, foi por ele prognosticado como incapaz de se beneficiar de qualquer intervenção educativa. Entretanto, o jovem médico Jean Itard, hoje considerado o precursor da educação especial, questionou as afirmações de Pinel e apostou nas potencialidades educativas de Victor, desenvolvendo um programa educativo com a ajuda do governo francês. Itard não conseguiu fazer com que Victor falasse, entretanto, contrariando o saber da época, conseguiu provar, com os primeiros resultados obtidos, que Victor não era um "imbecil sem esperança". Segundo Baptista e Oliveira (2002), o desenvolvimento das habilidades de Victor, a partir da convivência com outras pessoas, minimizava parte de uma limitação associada à sua educação: o isolamento social.

Da mesma forma, proporcionar às crianças com autismo oportunidades de conviver com outras da mesma faixa etária possibilita o estímulo às suas capacidades interativas, impedindo o isolamento contínuo. Além disso, subjacente ao conceito de competência social está a noção de que as habilidades sociais são passíveis de serem adquiridas pelas trocas que acontecem no processo de aprendizagem social. Entretanto, esse processo requer respeito às singularidades de cada criança. Diante dessas considerações, fica evidente que crianças com desenvolvimento típico fornecem, entre outros aspectos, modelos de interação para as crianças com autismo, ainda que a compreensão social destas últimas seja difícil. A oportunidade de interação com pares é a base para o seu desenvolvimento, como para o de qualquer outra criança. Desse modo, acredita-se que a convivência compartilhada da criança com autismo na escola, a partir da sua inclusão no ensino comum, possa oportunizar os contatos sociais e favorecer não 
só o seu desenvolvimento, mas o das outras crianças, na medida em que estas últimas convivam e aprendam com as diferenças. Em uma revisão da literatura sobre as diferentes intervenções que têm sido recentemente utilizadas no tratamento do autismo, Bosa (2006) destaca as evidências de que a provisão precoce de educação formal, a partir dos dois aos quatro anos, aliada à integração de todos os profissionais envolvidos, tem obtido bons resultados.

No entanto, conforme Tezzari e Baptista (2002), a possibilidade de inclusão de crianças deficientes lamentavelmente ainda está associada àquelas que não implicam uma forte reestruturação e adaptação da escola. Nesse sentido, crianças com prejuízos e déficits cognitivos acentuados, como psicóticos e autistas, não são consideradas em suas habilidades educativas (Baptista \& Oliveira, 2002). O fato de existirem poucos estudos sobre inclusão de crianças autistas na rede comum de ensino parece refletir esta realidade, isto é, a de que existem poucas crianças incluídas, se comparadas àquelas com outras deficiências. A literatura tem demonstrado que isso se deve, em grande parte, à falta de preparo de escolas e professores para atender à demanda da inclusão. Kristen, Brandt e Connie (2003) examinaram o relacionamento entre professores de escola comum e 12 alunos com autismo de segunda e terceira séries e observaram que, nos casos em que os professores percebiam mais positivamente seu relacionamento com os alunos com autismo, o índice de problemas de comportamento dessas crianças foi menor, e elas foram mais socialmente incluídas na sala de aula. Nesse sentido, a atuação junto ao professor é fundamental para que a inclusão escolar aconteça de forma satisfatória (Beyer, 2005). Sobre esse aspecto, Cacciari, Lima e Bernardi (2005) afirmam que, no processo de inclusão, a instituição e os professores demandam tanta atenção quanto a criança. De fato, Sant'Ana (2005) investigou, através de entrevistas, as concepções de 10 professores e 6 diretores de escolas públicas do Ensino Fundamental sobre a experiência de inclusão. Foram identificadas diversas dificuldades apontadas pelos professores, tais como a falta de orientação, estrutura e recursos pedagógicos. Além disso, foi verificado que os professores tendem a confundir os princípios de inclusão e integração. Embora em alguns países os vocábulos integração e inclusão sejam considerados sinônimos, no Brasil há uma tendência a diferenciá-los (Mantoan, 1998; Sassaki, 1998). Enquanto na integração investe-se na possibilidade de indivíduos com deficiência frequentarem escolas comuns de ensino, cujos currículo e método pedagógicos estão voltados para crianças consideradas "normais", na inclusão muda-se o foco do indivíduo para a escola. Neste caso, é o sistema educacional e social que deve adaptar-se para receber a criança deficiente.
Especificamente sobre a questão do autismo, em seu estudo, Jordan (2005) também aponta a necessidade de orientação aos professores, pois é a falta de conhecimento a respeito dos transtornos autísticos que os impede de identificar corretamente as necessidades de seus alunos com autismo. Na medida em que o sujeito é visto somente sob o ângulo de suas limitações, a crença na sua educabilidade e possibilidades de desenvolvimento estará associada à impossibilidade de permanência deste sujeito em espaços como o ensino comum.

De fato, um estudo exploratório sobre as expectativas dos professores frente à possibilidade de inclusão de alunos com autismo em suas classes (Goldberg, Pinheiro, \& Bosa, 2005) demonstrou que os professores manifestaram uma tendência a centralizar suas preocupações em fatores pessoais como, por exemplo, medo e ansiedade frente à sintomatologia mais do que à criança em si. $\mathrm{O}$ interessante é que entre os principais temores estava a dúvida em como lidar com a agressividade dos alunos. Esse resultado é intrigante, uma vez que a agressividade não é um comportamento prototípico de autismo, aparecendo em uma variedade de outras condições. Resultado semelhante foi obtido no estudo de Baptista, Vasques e Rublescki (2003) sobre a educação de crianças com transtornos de desenvolvimento, concluindo que muitos educadores resistem ao trabalho com crianças autistas devido a temores em não saber lidar com a agressividade delas - aliás, um aspecto que não é necessariamente característico desta condição.

Os resultados dos estudos sobre autismo demonstram que os professores apresentam ideias distorcidas a respeito do mesmo, principalmente quanto à (in) capacidade de comunicação. Não surpreendentemente, essas concepções parecem influenciar as práticas pedagógicas e as expectativas acerca da educabilidade desses alunos. As dificuldades dos professores, de um modo geral, se apresentaram na forma de ansiedade e conflito ao lidar com o "diferente". Por exemplo, no estudo de Goldberg, Pinheiro e Bosa (2005) houve a tendência de o professor adotar estratégias que, de certa forma, inibem a expressão dos "sintomas autistas" (e.g., "manter o aluno ocupado"). Tal estratégia era utilizada mais como uma tentativa de "dominar" a própria ansiedade do que como uma prática pedagógica, calcada nas necessidades do aluno. Ideias preconcebidas e caricaturizadas sobre o autismo, principalmente a partir da mídia, influenciam as expectativas do professor sobre o desempenho de seus alunos, afetando a eficácia de suas ações quanto à promoção de habilidades. $\mathrm{O}$ trabalho pedagógico integrador transforma limitações em um desafio para todos, através de uma postura de confiança na capacidade de mudança do aluno, em situações de confronto (Baptista, 2002). Alguns estudos têm demonstrado que, quando isso ocorre e os profes- 
sores estão adequadamente envolvidos no processo de inclusão, é possível verificar importantes ganhos para o desenvolvimento de crianças com autismo incluídas no ensino comum. Um exemplo disto é o estudo realizado por Serra (2004), no qual buscou verificar os efeitos da inclusão em escola comum nos comportamentos de um menino de sete anos com autismo. Os resultados obtidos através de uma avaliação da dinâmica familiar e escolar e da própria criança mostraram que a inclusão trouxe benefícios para ela. Por exemplo, a criança apresentou melhora significativa da concentração nas atividades propostas, bom estabelecimento de relacionamentos com os colegas e no comportamento de atender a ordens. Constataram-se, ainda, efeitos positivos da inclusão, na família, em função do maior investimento desta na aprendizagem da criança e um aumento na credibilidade nas potencialidades do filho. A partir da análise qualitativa de uma entrevista, outro estudo (Li, 2002) investigou a perspectiva de cinco pais de crianças com autismo quanto ao sucesso da inclusão escolar. Verificou-se que os pais observaram aumento de benefícios da inclusão na escola comum comparados aos da educação especial e o aumento das habilidades cognitivas, sociais e de comunicação, embora com dificuldades de generalização dessas habilidades para outros contextos. De forma similar, Yang, Wolfberg, Wu e Kwu (2003) verificaram em Taiwan notáveis ganhos na interação social recíproca e simbólica de crianças com autismo que participaram de jogos e brincadeiras em grupo, sendo que o convívio escolar proporcionou essas oportunidades. Por outro lado, segundo Chamberlain (2002), um processo de inclusão malsucedido pode aumentar os riscos de isolamento, rejeição dos pares e baixa qualidade de amizades. Em seu estudo, métodos de agrupamento de rede social foram utilizados para caracterizar o envolvimento de 14 crianças com autismo de "alto funcionamento" (sem deficiência mental associada) nas estruturas sociais da sala de aula de turmas de segunda a quarta séries. As medidas dos agrupamentos de pares indicaram que, embora as crianças com autismo não tenham sido ativamente rejeitadas, elas foram menos proeminentes que seus pares sem autismo na rede social e apresentaram menores níveis de aceitação, companhia e amizade recíproca que as demais crianças.

Sobre os efeitos de eventuais rejeições à criança com autismo por seus pares, um estudo envolvendo observações etnográficas de 16 crianças com autismo de alto funcionamento e idades entre 8 e 12 anos (Ochs, Kremer-Sadlik, Solomon, \& Sirota, 2001) indicou que elas demonstraram uma gama de reações à rejeição e desprezo, apesar das dificuldades na interpretação das intenções e sentimentos dos mesmos. Quando não há ambiente apropriado e condições adequadas à inclusão, a possibilidade de ganhos no desenvolvimento cede lugar ao prejuízo para todas as crianças. Isso aponta para a necessidade de reestruturação geral do sistema social e escolar para que a inclusão se efetive.

Desse modo, Karagiannis, Stainback e Stainback (1999) referem que, diante de uma inclusão adequada, mesmo que uma criança apresente deficiências cognitivas importantes e apresente dificuldades em relação aos conteúdos do currículo da educação comum, como pode ser o caso do autismo, ela pode beneficiar-se das experiências sociais. O objetivo do aprendizado de coisas simples do dia-a-dia (e.g., conhecer-se, estabelecer relações) seria o de as tornarem mais autônomas e independentes possíveis, podendo conquistar seu lugar na família, na escola e na sociedade. Desse modo, "na medida em que esses 'conteúdos' vão sendo desenvolvidos e 'aprendidos' por esses alunos, torna-se possível a entrada de outros conteúdos, da alfabetização, da matemática, etc." (Zilmer, 2003, p. 30). Com a educação de todas as crianças conjuntamente, aquelas que possuem alguma necessidade educativa especial, seja qual for, têm a oportunidade de preparar-se para a vida em comunidade, sendo que os professores melhoram suas habilidades profissionais e a sociedade funciona de acordo com o valor da igualdade de direitos para todas as pessoas. Em relação à inclusão de crianças com autismo, os estudos encontrados apontam os ganhos que essas crianças possuem diante das oportunidades de interação com pares em settings inclusivos. Entretanto, algumas limitações metodológicas como, por exemplo, a amostra selecionada e o delineamento utilizado ainda deixam lacunas relativas às suas potencialidades interativas e a sua possibilidade de inclusão no ensino comum, desde a educação infantil.

A revisão da literatura mostrou que, entre os raros estudos encontrados na área da psicologia sobre a inclusão escolar de crianças com autismo, o foco principal é a percepção de pais e professores quanto a esta possibilidade e nos seus efeitos familiares e escolares (Baptista et al., 2003; Goldberg, 2002; Goldberg, Pinheiro, \& Bosa, 2005; Kristen et al., 2003; Li, 2002). Este também tende a ser o foco em alguns estudos na área da educação, investigando, por exemplo, aspectos relacionados a questões institucionais de gestão escolar frente à inclusão (Rublescki, 2004) e à investigação das concepções de professores acerca de seu preparo para o trabalho com crianças autistas (Lira, 2004; Oliveira, 2002). Desse modo, tais estudos privilegiam a investigação das concepções de pais e professores, através de análise de conteúdo de entrevistas, sem investigar diretamente o comportamento social da criança na escola. Charman, Howlin, Berry e Prince (2004) demonstraram, através de entrevista com 125 pais de crianças com autismo (57 das quais foram refeitas após um ano de 
inclusão de seus filhos), os progressos no desenvolvimento de crianças, tais como mudanças positivas nas habilidades de socialização e comunicação.

Outros estudos investigaram as interações sociais de crianças autistas na escola, porém o foco principal foi o impacto de diferentes programas de intervenção, previamente estruturados. Owen-DeSchryver, Carr, Cale e Blakeley-Smith (2008) investigaram os efeitos da inclusão em crianças com autismo, de segunda e quarta séries, a partir da intervenção social. Para cada uma das três crianças com autismo investigadas havia de duas a quatro crianças com desenvolvimento típico instruídas em sessões de treinamento, que objetivaram o aumento das interações sociais entre elas e seus pares com autismo. Os dados coletados na hora do lanche das crianças na escola demonstraram aumento das iniciações interativas tanto dos pares treinados quanto das crianças com autismo. De forma semelhante, Kok, Kong e Bernard-Opitz (2002) compararam os efeitos de abordagens com jogos estruturados na comunicação e comportamento de jogo de crianças pré-escolares com autismo e seus pares. Foi demonstrado que elas apresentam maior frequência de comportamento de jogo e iniciações comunicativas nesse tipo de situação. Outro estudo envolvendo intervenção no contexto escolar foi realizado em Taiwan por Yang, 1-Iuang, Schaller e Tsai (2003). Eles compararam o efeito generalizado de um treinamento de habilidades socioemocionais em dois meninos e duas meninas com autismo, comparados a casos-controle, buscando promover o comportamento social no ambiente escolar de ensino comum. Os resultados da análise de regressão mostraram que todos os participantes do grupo experimental aumentaram a sequência de comportamentos sociais sobre o tempo, em relação ao grupo controle. Ainda sobre o comportamento social, Zercher, Hunt, Schuler e Webmaster (2001) investigaram os efeitos da participação em jogos integrados de dois irmãos gêmeos de 6 anos (autistas) comparados a três meninas com desenvolvimento típico de 5, 9 e 11 anos, respectivamente. Os resultados também indicaram que a participação de crianças autistas em jogos integrados com crianças com DT, programados e estruturados de acordo com o seu nível de desenvolvimento, produziu aumento significativo na atenção compartilhada de objetos, jogo simbólico e expressão verbal. Cabe ressaltar, no entanto, que o estudo não foi desenvolvido no ambiente escolar, e em algumas etapas do estudo houve a intervenção de um adulto com e sem coação das interações. O mesmo pode ser dito em relação ao estudo desenvolvido na Grécia (Gena, 2006) que encontrou aumento nas iniciações sociais com pares de quatro crianças pré-escolares com autismo, a partir do reforço de um professor. Embora tais estudos utilizem delineamentos e coletas de dados amplamente considerados, a observação direta dessas crianças no contexto de inclusão escolar e a avaliação da qualidade de suas interações com seus pares em uma abordagem desenvolvimentalista (Lampreia, 2007) é um aspecto metodológico importante, não encontrado na maioria dos estudos nesta área.

Uma das poucas exceções foi o estudo desenvolvido por Ochs et al. (2001) que, através da observação etnográfica, estudou o cotidiano das crianças autistas, no pátio e na sala de aula, e identificou as reações das mesmas frente aos sinais de rejeição dos pares. Entretanto, desconsiderou o comportamento manifesto das demais crianças em relação a elas, numa perspectiva interacionista. Do mesmo modo, Rotheram-Fuller (2006) estudou o comportamento social de 33 crianças do jardim de infância, segunda e quinta séries com seus pares típicos e comparou as diferenças entre os três níveis escolares, onde encontrou que as crianças mais jovens são menos rejeitadas, porém menos conectadas com seus pares que as crianças das séries mais avançadas. Nesse caso, o estudo não explora aspectos comparativos entre as crianças com autismo e seus pares com desenvolvimento típico.

Chamberlaim (2002), ao investigar a rede social e o envolvimento de crianças com autismo de alto funcionamento cognitivo e seus pares na escola, considerou apenas uma amostra de crianças de segunda a quarta séries. Portanto, como essa realidade ocorre em crianças menores (pré-escolares) e com deficiência mental associada, que representa a maioria dos casos de autismo, ainda é desconhecida. Percebe-se, sobretudo na área da psicologia, a carência de estudos relacionados à inclusão da criança autista em escola comum, focando a interação dessa criança com as demais e a caracterização de suas possíveis potencialidades interativas.

\section{Considerações Finais}

A análise dos poucos estudos na área de inclusão escolar e autismo apontam para a identificação de competência social nessas crianças, neste contexto. Entretanto, essa é uma habilidade que depende de um conjunto de medidas como, por exemplo, a qualificação dos professores, apoio e valorização do seu trabalho. Mostrar que a escola pode ser, de fato, um espaço de desenvolvimento da competência social para crianças autistas é ainda um grande desafio para os pesquisadores desta área.

Torna-se, portanto, de grande relevância novas pesquisas no campo da psicologia para atender a essa necessidade. São urgentes as investigações com crianças pré-escolares, visto que nessa época as crianças defrontam-se com as primeiras experiências com outras crianças, fora do contexto familiar e recreativo. De acordo com Barbosa (2007), a educação infantil se configura 
em um dos espaços contemporâneos de socialização das crianças desde tenra idade, permitindo às mesmas a interiorização de normas, valores, funções cognitivas, conhecimentos e práticas, pela convivência com novos sujeitos de interação. Dessa forma, a qualidade dessas primeiras experiências provavelmente influirá na adaptação social nos anos escolares subsequentes.

A minimização das crenças distorcidas sobre a (in)capacidade interativa das crianças autistas também pode ser conseguida através de delineamentos de pesquisa desenvolvidos com esta finalidade. Por exemplo, a utilização de "caso-controle" com crianças "típicas" em estudos observacionais permitiria não somente a identificação de áreas socialmente "deficitárias" na criança com autismo, mas em que aspectos ela se assemelha a outras crianças da mesma faixa etária, com desenvolvimento típico. Os estudos devem, portanto, focalizar não apenas a ocorrência de determinados comportamentos, mas a sua frequência e intensidade. Dessa forma, talvez seja possível mostrar que as diferenças nos comportamentos sociais sejam qualitativas e que mesmo a sua baixa frequência não é equivalente à sua ausência. Estudos que investiguem esses aspectos poderão contribuir para a dissolução de diversos mitos em torno da educabilidade de crianças com autismo, fornecendo evidências de que é possível o investimento em um espaço que, acima de tudo, é um direito.

\section{Nota}

${ }^{1}$ Agradecemos à Profa. Dra. Ana Almeida (Universidade do Minho, Portugal) pelas contribuições a este trabalho e à CAPES pelo apoio financeiro.

\section{Referências bibliográficas}

Almeida, A. (1997). As relações entre pares em idade escolar. Um estudo de avaliação da competência social pelo método $Q-$ sort. Tese de Doutorado, Universidade do Minho, Portugal.

Aranha, M. S. L. F. (1993). A interação social e o desenvolvimento humano. Temas em Psicologia, 3, 19-28.

Associação Psiquiátrica Americana (2002). Manual diagnóstico e estatístico de transtornos mentais. Porto Alegre: Artes Médicas

Baptista, C. R. (2002). Integração e autismo: análise de um percurso integrado. In C. R. Baptista \& C. A. Bosa (Orgs.), Autismo e educação: reflexões e propostas de intervenção (pp. 127-139). Porto Alegre: Artmed.

Baptista, C. R. \& Oliveira, A. C. (2002). Lobos e médicos: primórdios na educação dos "diferentes". In C. R. Baptista \& C. A. Bosa (Orgs.), Autismo e educação: reflexões e propostas de intervenção (pp. 93-109). Porto Alegre: Artmed.

Baptista, C. R., Vasques, C. K., \& Rublescki, A. F. (2003). Educação e transtornos globais do desenvolvimento: em busca de possibilidades. Cadernos da APPOA, 114, 31-36.

Barbosa, M. C. (2007). Culturas escolares, culturas de infância e culturas familiares: as socializações e a escolarização no entretecer destas culturas. Educação e Sociedade, 28, 1059-1083.

Beyer, H. O. (2005). Inclusão e avaliação na escola de alunos com necessidades educacionais especiais. Porto Alegre: Meditação.

Bosa, C. A. (2002). Autismo: atuais interpretações para antigas observações. In C. R. Baptista \& C. A. Bosa (Orgs.), Autismo e educação: reflexões e propostas de intervenção (pp. 21-39). Porto Alegre: Artmed.

Bosa, C. A. (2006). Autismo: intervenções psicoeducacionais. Revista Brasileira de Psiquiatria, 28, 47-53.

Bosa, C. A. \& Callias, M. (2000). Autismo: breve revisão de diferentes abordagens. Psicologia, Reflexão e Crítica, 13, $167-177$.

Cacciari, F. R., Lima, F. T., \& Bernardi, M. R. (2005). Ressignificando a prática: um caminho para a inclusão. Construção Psicopedagógica, 13, 13-28.

Cairns, R. B. (1986). A contemporary perspective on social development. In P. S. Strain, M. Guralnick, \& H. M. Walker (Orgs.), Children's social behavior. Development, assessment, and modification (pp. 3-47). Orlando: Academic Press.

Castro, R. E. F., Melo, M. H. S., \& Silvares, E. F. M. (2003). O julgamento de pares de crianças com dificuldades interativas após um modelo ampliado de intervenção. Psicologia: Reflexão e Crítica, 16, 309-318.

Chamberlain, B. O. (2002). Isolation or involvement? The social networks of children with Autism included in regular classes. Dissertation Abstracts International, 62 (8-A), 2680. (UMI No.AAI3024149)

Charman, T., Howlin, P., Berry, B., \& Prince, E. (2004). Measuring developmental progress of children with autism spectrum disorder on school entry using parent report. Autism, 8, 89-100.

Corsaro, W. A. (1997). The sociology of childhood. Thousand Oaks, CA: Pine Forge Press.

Del Prette, Z. A. P. \& Del Prette, A. (1996). Habilidades sociais: uma área em desenvolvimento. Psicologia Reflexão e Crítica, 9, $233-255$.

Del Prette, Z. A. P. \& Del Prette, A. (1999). Psicologia das habilidades sociais: terapia e educação. Petrópolis, RJ: Vozes.

Del Prette, Z. A. P. \& Del Prette, A. (2006). Avaliação multimodal de habilidades sociais em crianças: procedimentos, instrumentos e indicadores. In M. Bandeira, Z. A. P. Del Prette, \& A. Del Prette (Orgs.), Estudos sobre habilidades sociais e relacionamento interpessoal (pp. 47 - 68). São Paulo: Casa do Psicólogo.

Dessen, M. A. \& Aranha, M. S. L. F. (1994). Padrões de interação social nos contextos familiar e escolar: análise e reflexões sob a perspectiva do desenvolvimento. Temas em Psicologia, 3, $73-90$.

Dubar, C. (1999). La socialisation: Construction des identités sociales et professionnelle. Paris: A. Colin.

Farrington, D. P., Gallagher, B., Morley, L. St., Ledger, R. J., \& West, D. J. (1986). Unemployment, school leaving, and crime. British Journal of Criminology, 26, 335-356.

Gadia, C.; Tuchman, R., \& Rotta, N. (2004). Autismo e doenças invasivas do desenvolvimento. Jornal de Pediatria, 80, $583-594$.

Gena, A. (2006). The effects of prompting and social reinforcement on establishing social interactions with peers during the inclusion of four children with autism in preschool. International Journal of Psychology, 41(6), 541-554. 
Goldberg, K. (2002). A percepção do professor acerca do seu trabalho com crianças portadoras de autismo e síndrome de Down: um estudo comparativo. Dissertação de Mestrado, Universidade Federal do Rio Grande do Sul.

Goldberg, K., Pinheiro, L. R. S., \& Bosa, C. A. (2005). A opção do professor pela área de educação especial e sua visão acerca de um trabalho inclusivo. Perspectiva, 107, 59 - 68.

Gronlund, N. E. \& Holmlund, W. S. (1958). The value of elementary school sociometric status scores for predicting pupil's adjustment in high school. Educational Administration and Supervision, 44, 225-260.

Gupta, A. \& State, M. (2006). Autismo: genética. Revista Brasileira de Psiquiatria, 28, 29-38.

Hartup, W. W. (1983). Peer relations. In P. H. Mussen \& J. Carmichael (Orgs.), Handbook of child psychology: Vol. 4. Socialization, personality, and social development. (pp. 103-196). New York: John Wiley and Sons.

Hartup, W. W. (1989). Social relationships and their developmental significance. American Psychologist, 44, 120-126.

Hartup, W. W. (1992). Friendships and their developmental significance. In H. McGurk (Org.), Childhood social development (pp. 175-205). Gove: Erlbaum.

Hartup, W. W. (1996). The company they keep: Friendships and their developmental significance. Child Development, $67,1-13$.

Jordan, R. (2005). Managing Autism and Asperger's syndrome in current educational provision. Pediatric Rehabilittion, 8, $104-112$.

Kanner, L. (1943). Affective disturbances of affective contact. The Nervous Child, 2, 217 - 250.

Karagiannis, A., Stainback, S., \& Stainback, W. (1999). Fundamentos do ensino inclusivo. In S. Stainback \& W. Stainback (Orgs.), Inclusão - Um guia para educadores (M. Lopes, Trad., pp. 21-34). Porto Alegre: Artmed.

Klin, A. (2006). Autismo e síndrome de Asperger: uma visão geral. Revista Brasileira de Psiquiatria, 28, 3 - 11.

Kok, A. J., Kong, T. Y., \& Bernard-Opitz, V. (2002). A comparison of the effects of structured play and facilitated play approaches on preschoolers with autism: a case study. Autism, 6, $181-196$.

Kristen, R., Brandt, C., \& Connie, K. (2003). General education teachers' relationships with included students with Autism. Journal of Autism and developmental disorders, 33, 123-130.

Lampreia, C. (2007). A perspectiva desenvolvimentista para a intervenção precoce no autismo. Estudos de Psicologia, 24(1), 105-114.

Li, M. M. L. (2002). Factors leading to success in full inclusion placements for students with Autism. Dissertation Abstracts International, 63 (5-B) 2591. (UMI No. AAI3052995)

Lira, S. M. de (2004). Escolarização de alunos com transtorno autista: histórias de sala de aula. Dissertação de Mestrado, Universidade do Estado do Rio de Janeiro.

Lord, C. \& Magil, J. (1989). Methodological end theoretical issues in studying peer-directed behavior and Autism. In G. Dawson (Org.), Autism: Nature, diagnosis \& treatment. (p. 327-345). New York: Guilford.

Lordelo, E. R. \& Carvalho, A. M. A. (1998). Creche como contexto de desenvolvimento: parcerias adulto-criança e criançacriança. Temas de Psicologia, Ribeirão Preto, 6, 117-128.

Mantoan, M. T. E. (1998). Integração x Inclusão - Educação para todos. Pátio, 5, 48 - 51.
Mead, G. H. (1972). Mind, self and society: From the standpoint of a social behaviorist. Chicago: University of Chicago (Trabalho original publicado em 1934).

Moura, M. A. S. (1993). A interação social e solução de problemas por crianças: questões metodológicas, resultados empíricos e implicações educacionais. Temas em Psicologia, 3, $39-47$.

Ochs, E., Kremer-Sadlik, T., Solomon, O., \& Sirota, K. G. (2001). Inclusion as social practice: Views of children with Autism. Social Development. 10, 399 - 419.

Oliveira, A. C. (2002). O autismo e as "crianças-selvagens": da prática da exposição às possibilidades educativas. Dissertação de Mestrado, Universidade Federal do Rio Grande do Sul.

Oliveira, Z. M. R. \& Rossetti-Ferreira, M. C. (1993). O valor da interação criança-criança em creches no desenvolvimento infantil. Cadernos de Pesquisa, 87, 62-70.

Olwes, D. (1980). Familial and temperamental determinants of aggression behavior in adolescents - A causal analysis. Developmental Psychology, 16, 644 - 660.

Owen-DeSchryver, J. S., Carr, E. G., Cale, S. I., \& BlakeleySmith, A. (2008).Promoting social interactions between students with autism spectrum disorders and their peers in inclusive school settings. Focus on Autism and Other Developmental Disabilities, 23(1), 15-28.

Parker, J. G. \& Asher, S. R. (1987). Peer relationships and later personal adjustment: Are low-accepted children at risk? Psychological Bulletin, 102, 357 - 389.

Pedrosa, M. \& Carvalho, A. M. A. (2005). Análise qualitativa de episódios de interação: uma reflexão sobre procedimentos e formas de uso. Psicologia Reflexão e Crítica, 18, 431 - 442.

Pepler, D. J. \& Craig, W. M. (1998). Assessing children's peer relationsships. Child Psychology \& Psychiatry Review, 3, $176-182$.

Rotheram-Fuller, E. J. (2006). Age-related changes in the social inclusion of children with autism in general education classrooms. Dissertation Abstracts International, 66 (7-A), 2493. (UMI No. AAI3181739)

Rublescki, A. F. (2004). A caminho da escola... Um estudo sobre a educação integrada de crianças com autismo e psicose infantil. Dissertação de Mestrado, Universidade Federal do Rio Grande do Sul.

Sant'Ana, I. M. (2005). Educação inclusiva: concepções de professores e diretores. Psicologia em Estudo, 10, 227 - 234.

Sassaki, R. K. (1998). Integração e inclusão: do que estamos falando? Temas sobre desenvolvimento, 39, 45 - 47.

Serra, D. C. G. (2004). A inclusão de uma criança com autismo na escola regular: desafios e processos. Dissertação de Mestrado, Universidade do Estado do Rio de Janeiro.

Shaffer, H. R. (1996). Social development. Cambridge: Blackwell Publisher.

Tezzari, M. \& Baptista, C. R. (2002). Vamos brincar de Giovani? A integração escolar e o desafio da psicose. In C. R. Baptista \& C. A. Bosa (Orgs.), Autismo e educação: reflexões e proposta de intervenção (pp.145-156). Porto Alegre: ArtMed.

Volkmar, F., Lord, C., Bailey, A., Schultz, R. \& Klin, A. (2004). Autism and pervasive developmental disorders. Journal of Child Psychology and Psychiatry, 45, 135 - 170.

Waters, E. \& Sroufe, L. A. (1983). Social competence as a developmental construct. Developmental Review, 3, 79 - 97.

Yang, N. K., 1-Iuang, T., Schaller, J. L., \& Tsai, M. H. W. S. (2003). Enhancing appropriate social behaviors for children with autism in general education classrooms: An analysis of 
six cases. Education and Training in Developmental Disabilities, 38(4), 405-416.

Yang, T. R., Wolfberg, P. J., Wu, S. C., \& Hwu, P. Y. (2003). Supporting children on the autism spectrum in peer play at home and school: Piloting the integrated play groups model in Taiwan. Autism, 7, $437-453$.

Zercher, C., Hunt, P., Schuler, A., \& Webmaster, J. (2001). Increasing joint attention, play and language trough peer supported play. Autism, 5, 374-398.

Zilmer P. (2003). Reflexões sobre a prática: Escola ou clínica? In M. S. Charczuk \& M. N. Folberg (Orgs.), Crianças psicóticas e autistas: a construção de uma escola (pp. 27-38). Porto Alegre: Mediação.

Síglia Pimentel Höher Camargo é Psicóloga pela UFSM e Mestre em Psicologia do Desenvolvimento pela UFRGS.

Endereço para correspondência: Instituto de Psicologia

-UFRGS. Rua Ramiro Bracelos 2600, sala 110. Bairro

Santa Cecília. Porto Alegre-RS. CEP:90035-003.

E-mail: sigliahoher@yahoo.com.br

Cleonice Alves Bosa é Profa. Dra. do Programa de Pósgraduação em Psicologia da UFRGS.

Endereço para correspondência: Instituto de Psicologia

- UFRGS. Rua Ramiro Barcellos, 2600, Sala 110. Bairo

Santa Cecília - Porto Alegre-RS. CEP: 90035-003.

E-mail: cleobosa@uol.com.br

Competência social, inclusão escolar e autismo: revisão crítica da literatura

Síglia Pimentel Höher Camargo e Cleonice Alves Bosa

Recebido: 26/02/2008

$1^{\text {a }}$. Revisão: 09/06/2008

Aceite final: 13/06/2008 